\title{
Polyelectrolytes between two membranes or colloidal particles
}

\author{
J H J van Opheusden \\ Center for Theoretical Physics, University of Twente, POB 217, 7500 AE Enschede, The \\ Netherlands
}

Received 9 October 1987, in final form 1 February 1988

\begin{abstract}
We investigate the equilibrium behaviour of charged polymers of finite length in a Debye-screened potential between two parallel charged walls. For the polymer we take the continuum approximation to calculate the spectrum of eigenfunctions for the partition function. For finite but long polymers only the lowest two terms in this expansion contribute to first order to the configurational sum, corresponding to a symmetrical and an antisymmetrical solution. Within this formalism we calculate the monomer density distribution for finite strands of polymer attached to one or both surfaces (tails, loops and bridges) as well as for polymers free in solution. For the free polymer we also find the free energy as a function of the distance between the plates for several values of the interaction parameters, as well as the effective interaction between the plates due to the polyions. This shows that there can exist an equilibrium separation distance between colloidal particles due to the interactions with charged polymers in solution. This mechanism may also explain the formation of rouleaux for red blood cells.
\end{abstract}

\section{Introduction}

In this paper we will discuss the equilibrium density distribution of polyelectrolytes near red blood cells or charged colloidal particles when two of these come close together. As a simplified model we consider two parallel planes with, between the plates, an ionic fluid containing in addition to the small salt ions a small amount of polyelectrolyte. This can improve our understanding of the behaviour of polyelectrolytes in the small gap between two cells or colloidal particles when these are very close. When the polymer binds to the wall of the particles it can form bridges across the gap and thereby give rise to a very strong binding force between the particles. This is one mechanism to explain the formation of so-called rouleaux of red blood cells (Wiegel and Perelson 1981). For short-range interactions of the monomers with the surface of the particles other types of models can be considered, like the lattice model we have investigated earlier (van Opheusden et al 1985). In this model also a strong binding can occur.

\section{The Poisson-Boltzmann equation in one dimension}

For the Debye screened electrostatic field of a charged colloidal particle or a red blood cell very close to its surface it seems justified to consider a one-dimensional approximation. The surface is modelled as a single charged wall at $x=0$, extending to infinity in the $y$ and $z$ directions. In this particular case the full non-linear Poisson-Boltzmann 
equation can be solved (Lampert and Crandall 1980, Verwey and Overbeek 1948). The difference between this solution and that of the linearised equations is that there is somewhat stronger screening very close to the wall, while at larger distances the effects of non-linearity diminish rapidly. In principle there is no problem in investigating the behaviour of the polyelectrolytes in the potential that is the solution of the non-linear Poisson-Boltzmann equation. This term, when included in the equation for the partition function of the polyions, only plays the role of an external potential and the resulting equation remains completely linear. The only point is that the equation cannot simply be transformed into one for which solutions can be expressed in terms of elementary functions, as is the case for the formalism to be described next. One would have to rely instead on a numerical solution of the equations under consideration. One should note, moreover, that the screening effect is caused by the small ions, the polyions themselves not influencing the potential field. We will hence neglect all non-linear effects and consider the general solution of the linearised equation

$$
V(x)=A \exp \left(-k_{\mathrm{D}} x\right)+B \exp \left(k_{\mathrm{D}} x\right) .
$$

When the wall is held at a constant potential $V_{0}$ the bounded solution is

$$
V(x)=V_{0} \exp \left(-k_{\mathrm{D}} x\right)
$$

We want to calculate the field in the gap between two colloidal particles. Therefore we introduce a second wall, which is parallel to the first, and at a distance $\xi$ such that the potential $V_{0}$ is effectively screened, i.e. $\xi \gg k_{\mathrm{D}}^{-1}$. Raising the potential on the second wall to $V_{0}$ will then result in a redistribution of the ions that gives the same screening effect. The potential between two walls prepared in this way, located at positions $x=d$ and $x=-d$ is given by

$$
V(x)=V_{0} \frac{\cosh \left(k_{\mathrm{D}} x\right)}{\cosh \left(k_{\mathrm{D}} d\right)}
$$

To illustrate the validity of this approximation consider two spheres of radius $R$, far apart from each other, and each with a surface potential $V_{0}$. The Debye screened field of a single sphere at the orgin is

$$
V(r)=\frac{Q}{4 \pi \varepsilon r} \exp \left[k_{\mathrm{D}}(R-r)\right]
$$

In terms of the distance $\delta$ to the surface of the sphere, $\delta=r-R$, equation (4) becomes

$$
V(R+\delta)=V_{0} \exp \left(-k_{\mathrm{D}} \delta\right) R /(R+\delta) .
$$

Thus close to the surface the potential is approximated by that of the flat wall (equation (2)), as could be expected. If two spheres are placed along the $x$ axis, with their surfaces at $x=d$ and $x=-d$, respectively, the potential in the gap between the spheres along the $x$ axis becomes

$$
V(x)=2 V_{0} \exp \left(-k_{\mathrm{D}} d\right) \cosh \left(k_{\mathrm{D}} x\right) \quad(d \ll R)
$$

which is essentially the same solution as equation (3). This solution follows from a simple superposition of the potentials from both walls, which is allowed in the linearised theory. Note that we have assumed that the charge distribution on the colloidal particle itself does not change because of the other particle. 


\section{Small concentration of polyelectrolytes}

When a small number of polyelectrolyte chain molecules, with charge opposite to the colloidal particles, is added to the solution these chains will tend to adhere to the particles because of the attractive potential, while on the other hand the heat motion tends to move the chains away from the surface. In this section we calculate the partition function of a single, long but finite chain between two colloidal charged particles. For the adsorption to a single colloidal particle a continuous model has been used by Wiegel (1977). We will consider the effect of a second particle within this model.

We assume that the amount of polyelectrolyte is so small that it will not affect the charge distribution in the solution. Because we consider this low-density limit we may also ignore excluded volume effects between the polymers. The potential between the spheres is taken to be as given in equation (3). For chains of length $N$ the partition function $G\left(r_{0}, r_{N}, N\right)$ in the gap between the particles is the solution of the diffusionlike equation (Wiegel 1975, Freed 1972)

$$
\left[\partial / \partial N-\frac{1}{6} l^{2} \nabla^{2}+\beta V(r)\right] G\left(r_{0}, r_{N}, N\right)=0 .
$$

Here $r_{0}$ and $r_{N}$ are the positions of the particles at the two ends of the chain and $l$ is the length of one monomeric unit. Note again that the potential is not influenced by the charges on the polyelectrolytes themselves but only by the small counterions in solution. In particular, this implies that there is no extra swelling of the polyions, other than possibly a somewhat larger effective Kuhn length $l$. For the dilute solution of polyelectrolytes we neglect the small non-linearities this would produce in (7), which would otherwise have to be solved in a self-consistent fashion. For the parallel-plate geometry, equation (7) separates in Euclidean coordinates. The solution is a formal expansion in terms of eigenfunctions $\psi_{n}$ for the $x$ coordinates, and normalised Gaussians $n(\xi)$ in the $y$ and $z$ directions

$$
G\left(r_{0}, r_{N}, N\right)=n\left(y_{N}-y_{0}\right) n\left(z_{N}-z_{0}\right) \sum_{0}^{\infty} \psi_{n}\left(x_{N}\right) \psi_{n}^{*}\left(x_{0}\right) \exp \left(-\lambda_{n} N\right)
$$

where

$$
n(\xi)=(2 \pi \sigma)^{-1 / 2} \exp \left(-\xi^{2} / \sigma^{2}\right)
$$

The $\psi_{n}$ are the solutions of

$$
\left(-\frac{l^{2}}{6} \frac{\mathrm{d}^{2}}{\mathrm{~d} x^{2}}+\beta V(x)\right) \psi_{n}=\lambda_{n} \psi_{n}
$$

We must solve this equation in order to find the spectrum $\psi_{n}$ with corresponding $\lambda_{n}$, using expression (3) for the potential $V(x)$.

\section{Debye screened potential between two walls}

The problem of the adsorption of polyelectrolyte to a single wall with a Debye screened potential has been solved before (Wiegel 1977). For the single wall the potential is of the form (2)

$$
V(x)=-V_{0} \exp \left(-k_{\mathrm{D}} x\right)
$$


where we have introduced the extra minus sign to have an attractive potential, while the parameter $V_{0}$ remains positive. It was shown by Wiegel (1977) that a bound state exists provided

$$
24 \beta V_{0} / k_{\mathrm{D}}^{2} l^{2}>j_{0,1}^{2}
$$

with $j_{0,1}=2.4048 \ldots$ the first real zero of the Bessel function $J_{0}$. This solution can be found by considering equation (9) with the potential as given in (10) and making the substitution

$$
s(x)=\left(24 \beta V_{0} / k_{\mathrm{D}}^{2} l^{2}\right)^{-1 / 2} \exp \left(-\frac{1}{2} k_{\mathrm{D}} x\right) \equiv s_{0} \exp \left(-\frac{1}{2} k_{\mathrm{D}} x\right) .
$$

One then finds the Bessel equation

$$
\left(s^{2} \mathrm{~d}^{2} / \mathrm{d} s^{2}+s \mathrm{~d} / \mathrm{d} s+s^{2}+\Lambda_{n}\right) f_{n}(s)=0
$$

with

$$
\Lambda_{n}=24 \lambda_{n} \beta V_{0} / k_{\mathrm{D}}^{2} l^{2} \text {. }
$$

As boundary conditions we take $\psi(0)=0$ and $\psi(L)=0$ for some finite value of $L$, and consider the limiting solution as $L \rightarrow \infty$. If we now take the potential of both walls, equation (3), but with $x$ shifted over $L$, such that the first wall lies at $x=0$ and the second at $x=2 L$, we have

$$
V=-\frac{V_{0} \exp \left(k_{\mathrm{D}} L\right)}{2 \cosh \left(k_{\mathrm{D}} L\right)}\left[\exp \left(-k_{\mathrm{D}} x\right)+\exp \left(k_{\mathrm{D}} x\right) \exp \left(-2 k_{\mathrm{D}} L\right)\right]
$$

For $k_{\mathrm{D}} \ll L$, i.e. when the Debye screening length is small compared to the gap width, some simplifications are possible. The prefactor in this case is nearly equal to $V_{0}$, and the substitution (12) leads to an equation similar to equation (13):

$$
\left[s^{2} \mathrm{~d}^{2} / \mathrm{d} s^{2}+s \mathrm{~d} / \mathrm{d} s+s^{2}+s_{0}^{4} \exp \left(-2 k_{\mathrm{D}} L\right) / s^{2}+\Lambda_{n}\right] f_{n}(s)=0 .
$$

For $x<L$ the extra term can be considered to be a small perturbation, so we may use the solutions of the single-wall problem, though one must be careful with the boundary conditions. For the single wall the boundary condition at $x=L$ is $f\left(s_{L}\right)=0$, because one wants the solution to vanish when $L \rightarrow \infty$. Here we have used the shorthand notation

$$
s_{L}=s_{0} \exp \left(-k_{\mathrm{D}} L\right) .
$$

The effect of the second wall is that the potential for $x>L$ is exactly the mirror image of that for $x<L$. Hence the solutions of the eigenfunction equation will also have this symmetry, but there may be a parity change. In other words, next to the antisymmetric solutions, which have a nodal point at $x=L$, there exist symmetric solutions. In both cases the boundary condition at the impenetrable wall is a vanishing partition function, so we have

$$
f_{\mathrm{s}}\left(s_{0}\right)=0 \quad\left(\mathrm{~d} f_{\mathrm{s}} / \mathrm{d} s\right)_{s=s_{L}}=0
$$

for the symmetric solutions and

$$
f_{\mathrm{a}}\left(s_{0}\right)=0 \quad f_{\mathrm{a}}\left(s_{\mathrm{L}}\right)=0
$$

for the antisymmetric solutions. The general solution of the unperturbed equation is

$$
f(s)=C_{1} J_{\nu}(s)+C_{2} J_{-\nu}(s)
$$


with $\Lambda_{n}=-\nu^{2}$, and $J_{\nu}$ the Bessel function of order $\nu$. Substitution of the general solution into the boundary equation for the antisymmetric solution gives the equation

$$
J_{\nu}\left(s_{0}\right) / J_{-\nu}\left(s_{0}\right)=J_{\nu}\left(s_{L}\right) / J_{-\nu}\left(s_{L}\right)
$$

In the limit $L \rightarrow \infty$, that of the single wall, the right-hand side of this equation vanishes because $s_{L} \rightarrow 0$ and thus $J_{\nu}\left(s_{L}\right) \rightarrow 0$ for positive $\nu$. The boundary conditions for the symmetric solutions yield the relation

$$
\frac{J_{\nu}\left(s_{0}\right)}{J_{-\nu}\left(s_{0}\right)}=\frac{J_{\nu-1}\left(s_{L}\right)-J_{\nu+1}\left(s_{L}\right)}{J_{1-\nu}\left(s_{L}\right)-J_{-\nu-1}\left(s_{L}\right)}
$$

As is to be expected for the case $s_{L} \rightarrow 0$ both equations become equivalent. Strictly speaking the general solution is not valid at integer values of $\nu$, where $J_{\nu}$ and $J_{-\nu}$ are linearly dependent and other types of Bessel functions should be used. The distinction is not really important for the generic case of non-integer $\nu$. As stated for the single wall, the right-hand side vanishes and bound-state solutions exist only when $s_{0}>j_{0,1}$, the first zero of $J_{0}$. For any positive $\nu$ the first zero is increasing monotonically with $\nu$ (Jahnke et al 1966).

Of course one can solve equations (20) and (21) numerically. However, as we consider the case in which the two walls are not too close, we can do better. At least for the lowest bound state, when $s_{0}$ is between $j_{0,1}$ and $j_{0,2}=5.13562 \ldots$, we know that $s_{L}$ is also small. Hence we may use the asymptotic formula for the Bessel function with small argument (Abramowitz and Stegun 1965)

$$
J_{\nu}\left(s_{L}\right) \approx\left(\frac{1}{2} s_{L}\right)^{\nu} / \Gamma(1+\nu)
$$

Substituting this in (20) and (21), and using expression (17) for $s_{L}$, one obtains

$$
J_{\nu}\left(s_{0}\right)= \pm\left(\frac{1}{2} s_{0}\right)^{2 \nu} \exp \left(-\nu k_{\mathrm{D}} L\right) \frac{\Gamma(1-\nu)}{\Gamma(1+\nu)} J_{-\nu}\left(s_{0}\right)
$$

with the plus sign for the antisymmetric solutions and the minus sign for the symmetric ones. In the limit $L \rightarrow \infty$ the single solution is $\nu=\nu_{0}$ and for large but finite $L$ this level splits up into a doublet at $\nu_{ \pm} \approx \nu_{0} \pm \Delta \nu$ with

$$
\Delta \nu=\left(\frac{\partial J_{\nu}\left(s_{0}\right)}{\partial \nu}\right)_{\nu=\nu_{0}}^{-1}\left(\frac{1}{2} s_{0}\right)^{2 \nu_{0}} \exp \left(-\nu_{0} k_{\mathrm{D}} L\right) \frac{\Gamma\left(1-\nu_{0}\right)}{\Gamma\left(1+\nu_{0}\right)} J_{-\nu_{0}}\left(s_{0}\right)
$$

The splitting is important because it gives an indication about the relative importance of the antisymmetric solution in the eigenfunction expansion (8), as the eigenvalues are still given by $\Lambda=-\nu^{2}$ for both types. The relative weight of the antisymmetric solution for a polymer of length $N$ is approximately $\exp \left(-4 N \nu_{0} \Delta \nu\right)$. In the limit of very long chains, $N \gg 1$, the partition function is dominated by the lowest eigenvalue $\Lambda_{+}=-\nu_{+}^{2}$. For the double well we have to be a bit more careful. The lowest eigenvalue always corresponds to a symmetric solution, but the lowest antisymmetric solution has an eigenvalue relatively close to this one. Consequently, for quite large $N$, regardless of the symmetry of the problem itself, the solutions can be quite asymmetric, corresponding to a considerably higher concentration of polymer in one of the wells as compared to the other. 


\section{Monomer density between two walls}

By solving (20) and (21) we obtain the ground state and the first excited state of (13), with boundary conditions as given by $(18 a)$ and $(18 b)$. For the case of a long but finite polyelectrolyte these two solutions give the lowest two terms in the eigenfunction expansion for the partition function. We may neglect higher-order terms in this expansion because the corresponding eigenvalues $\lambda_{n}$ are separated from the lowest two by a relatively large energy gap. For infinite chain length the parition function is completely dominated by the ground-state solution.

In order to determine the density of polymer material at a certain point $x$ between the walls one has to consider all possible configurations of a polyelectrolyte chain which pass through this point. The number of configurations with endpoints at $x_{0}$ and $x_{N}$, and the $n$th monomer held fixed at point $x$, is proportional to the three-point function $G_{3}\left(x_{0}, x, x_{N}, n, N\right)$. As we consider a chain of the Markov type, without excluded volume effects, we can take this single chain to consist of two shorter chains, or two tails fixed together at position $x$. Both tails assume configurations according to the partition function of a polyelectrolyte of that specific length. Hence the density for all monomers becomes

$$
\rho(x)=\frac{C}{N} \sum_{n=0}^{N} \frac{\int \mathrm{d} x_{0} \int \mathrm{d} x_{N} G\left(x_{0}, x, n\right) G\left(x, x_{N}, N-n\right)}{\int \mathrm{d} x_{0} \int \mathrm{d} x_{N} G\left(x_{0}, x_{N}, N\right)}
$$

with $C$ a normalisation constant such that

$$
\int \mathrm{d} x \rho(x)=1
$$

Because we have a linear theory any overall constant can be used as a normalisation for the density function.

It is assumed implicitly in this equation that the endpoints of the polymer can move freely through the potential field within the gap, and all possible end positions have to be considered. For polymers attached to the surface with one end, the integration over $x_{0}$ in equation (25) is not appropriate, but instead one should consider the limiting behaviour when the endpoint approaches the surface. In this case

$$
\rho(x)=\frac{C}{N} \sum_{n=0}^{N} \lim _{x_{0} \rightarrow 0} \frac{\int \mathrm{d} x_{N} G\left(x_{0}, x, n\right) G\left(x, x_{N}, N-n\right)}{\int \mathrm{d} x_{N} G\left(x_{0}, x_{N}, N\right)} .
$$

An analogous expression holds for polymer loops, which are bound on both ends.

Strictly speaking, the approximation of $G$ by taking only the lower states is valid only for large polymer length, i.e. when the monomer considered is far from both ends. For long polymer chains the end effects are not very important, and one can use only the lowest, or two lowest terms, in the series expansion for the partition function

$$
G\left(x_{0}, x_{N}, N\right)=\sum_{k=0}^{\infty} \psi_{k}\left(x_{N}\right) \psi_{k}^{*}\left(x_{0}\right) \exp \left(-\lambda_{k} N\right)
$$

where the $\psi_{k}(x)$ are orthonormal eigenfunctions when written in the $s$ language, with a corresponding norm of the linear equation (13). In the case of ground-state dominance all end effects become irrelevant and both (25) and (27) reduce to

$$
\rho(x)=\psi_{0}^{2}(x)
$$


When there is no ground-state dominance matters become more complicated, even if one only considers the effect of the first excited state. In this case we have the ground-state doublet, formed by the symmetric solution $\psi_{\mathrm{s}}$ and the antisymmetric solution $\psi_{\mathrm{a}}$, which has a slightly higher eigenvalue. The difference $\Delta \lambda=\lambda_{\mathrm{a}}-\lambda_{\mathrm{s}}$ is small compared to the difference between $\lambda_{a}$ and the first excited symmetric state. The partition function becomes

$$
G\left(x_{1}, x_{2}, n\right)=\psi_{\mathrm{s}}\left(x_{1}\right) \psi_{\mathrm{s}}\left(x_{2}\right)+\psi_{\mathrm{a}}\left(x_{1}\right) \psi_{\mathrm{a}}\left(x_{2}\right) \exp (-n \Delta \lambda)
$$

where an overall constant $\exp \left(-\lambda_{\mathrm{s}} n\right)$ is neglected. When this is substituted in (25) the summation over $n$ can be performed, and the general result is, for large $N$ and small $\Delta \lambda$,

$$
\begin{aligned}
\rho(x)=\left(\iint \mathrm{d}\right. & x_{0} \mathrm{~d} x_{N}\left\{\psi_{\mathrm{s}}\left(x_{0}\right) \psi_{\mathrm{s}}\left(x_{N}\right) \psi_{\mathrm{s}}^{2}+\psi_{\mathrm{a}}\left(x_{0}\right) \psi_{\mathrm{a}}\left(x_{N}\right) \psi_{\mathrm{a}}^{2} \exp (-N \Delta \lambda)\right. \\
& \left.\left.+\left(\psi_{\mathrm{s}}\left(x_{0}\right) \psi_{\mathrm{a}}\left(x_{N}\right)+\psi_{\mathrm{a}}\left(x_{0}\right) \psi_{\mathrm{s}}\left(x_{N}\right)\right) \psi_{\mathrm{a}} \psi_{\mathrm{s}}[1-\exp (-N \Delta \lambda)] / N \Delta \lambda\right\}\right) \\
& \times\left(\iint \mathrm{d} x_{0} \mathrm{~d} x_{N}\left[\psi_{\mathrm{s}}\left(x_{0}\right) \psi_{\mathrm{s}}\left(x_{N}\right)+\psi_{\mathrm{a}}\left(x_{0}\right) \psi_{\mathrm{a}}\left(x_{N}\right) \exp (-N \Delta \lambda)\right]\right)^{-1}
\end{aligned}
$$

Here we have not explicitly written the $x$ dependence of the functions $\psi_{\mathrm{s}}$ and $\psi_{\mathrm{a}}$. Once these functions are known it is straightforward to perform the integrations in this equation. In fact we do not need the functions explicitly, because the integral over $\psi_{\mathrm{a}}$ is always identically zero.

For the free polymer chain, equation (24) gives immediately

$$
\rho_{\text {free }}(x)=\psi_{\mathrm{s}}^{2}(x)
$$

i.e. we get the same result as for ground-state dominance. For the polymer with both ends free in this order there is no effect on the density distribution between the particles. Higher-order terms in the eigenfunction expansion have to be included to find a deviation from the ground-state behaviour for shorter polymers. When one or both ends of the polymer are attached to the surface we have to be a little bit careful. As the functions $\psi_{\mathrm{s}}$ and $\psi_{\mathrm{a}}$ vanish at the surface we must consider the limiting behaviour as described for (27). For a polymer chain with one end attached to one surface and the other end attached to the other surface, forming a bridge between the particles, we have $\psi_{\mathrm{s}}\left(x_{0}+\delta\right)=\psi_{\mathrm{s}}\left(x_{N}-\delta\right)=S \varepsilon$ and $\psi_{\mathrm{a}}\left(x_{0}+\delta\right)=-\psi_{\mathrm{a}}\left(x_{N}-\delta\right)=A \varepsilon$, for $x_{0}=0$ and $x_{N}=2 L$, with $\varepsilon, \delta \rightarrow 0$. In other words we define $S=\psi_{\mathrm{s}}^{\prime}\left(x_{0}\right)$ and $A=\psi_{\mathrm{a}}^{\prime}\left(x_{0}\right)$. The density distribution for a bridge becomes

$$
\rho_{\text {bridge }}(x)=\left[S^{2} \psi_{\mathrm{s}}^{2}-A^{2} \psi_{\mathrm{a}}^{2} \exp (-N \Delta \lambda)\right] /\left[S^{2}-A^{2} \exp (-N \Delta \lambda)\right]
$$

which does show some effect of the antisymmetric solution. Relative to the solution for the finite chain with both ends free the density in the middle between the walls is increased. This comes from the fact that for the free chain many configurations exist in which the full chain is in one of the potential wells, and no monomer density in the centre is generated. All these configurations are excluded for the bridge model. Hence, by forcing the chain to cross from one wall to the other the relative abundance of this type of configuration in the total configuration sum is enhanced, and the density in the centre is increased.

When only one end is attached to a surface we have a polymer tail

$$
\rho_{\text {tail }}(x)=\left\{S \psi_{\mathrm{s}}^{2}+A \psi_{\mathrm{s}} \psi_{\mathrm{a}}[1-\exp (-N \Delta \lambda)] / N \Delta \lambda\right\} / S
$$


and for both ends attached to the same surface, a polymer loop, we find

$$
\begin{aligned}
& \rho_{\text {loop }}(x)=\left\{S^{2} \psi_{\mathrm{s}}^{2}+A^{2} \psi_{\mathrm{a}}^{2} \exp (-N \Delta \lambda)\right. \\
& \left.+2 A S \psi_{\mathrm{s}} \psi_{\mathrm{a}}[1-\exp (-N \Delta \lambda)] / N \Delta \lambda\right\} /\left[S^{2}+A^{2} \exp (-N \Delta \lambda)\right] .
\end{aligned}
$$

Note that the asymmetry for tails is approximately half the effect found for loops, as can be expected when one end is free to cross over to the other surface. When one considers the case in which there are polymer tails on both surfaces the asymmetry in (34) cancels completely, and the result for ground-state dominance is recovered, at least to this order. This is in accordance with the remark made above about the increase in density for polymer bridges near the centre; for polymer tails no such effect should be expected.

In figure 1 we plot the monomer density of bridges between the walls for a number of different values of the polymer length. One observes that for decreasing $N$ the density in the centre between the particles or cells increases, while it decreases accordingly near the walls. Because the symmetric solution $\psi_{\mathrm{s}}$ and the antisymmetric solution $\psi_{\mathrm{a}}$ that enter the equation for the monomer density of bridges (equation (33)) do not depend on the polymer length, all curves trivially pass through the point where $\psi_{\mathrm{a}}=\psi_{\mathrm{s}}$. In figure 2 have we plotted the density for tails, polyelectrolyte chains fixed to the wall at $x=0$. For increasing chain length one observes a decreasing asymmetry of the monomer density between the walls. For the case of infinite chain length, as pointed out, the density profiles of tails and bridges are, of course, identical.

In figures 3 and 4 one finds the monomer density of the infinite chain for various values of the depth of the potential well, or rather the parameter $s_{0}$ as defined in equation (12), and the distance between the walls in reduced units $k_{\mathrm{D}} L$, respectively. For decreasing values of $s_{0}$ one observes an increase of the density in the centre between

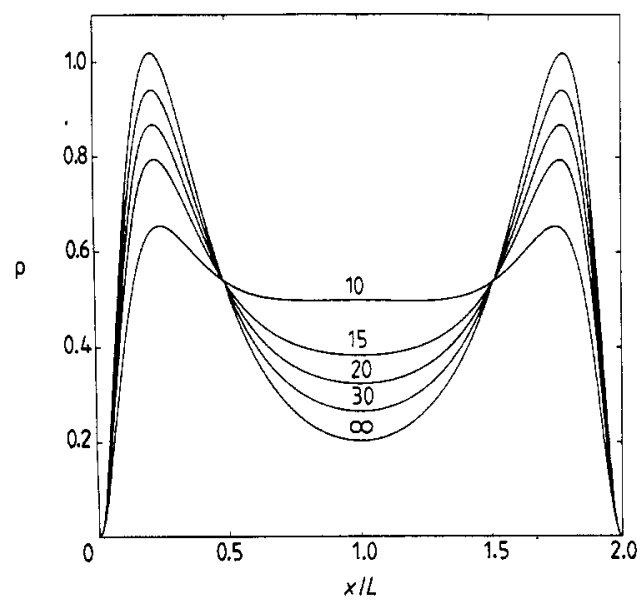

Figure 1. Monomer density between colloidal particles or cells with a Debye screened potential for bridges of various length. The polymers are fixed with one end to one particle, at $x=0$, while the other end is fixed to the surface at the other particle at $x / L=2$. The values of the parameters as used in the text are $s_{0}=3, k_{\mathrm{D}} L=10$ and the different lengths are given by $N=10,15,20,30$ and $\infty$, the limit of infinite chain length. One observes that for longer chains the polymer material is more confined in the potential wells near the wall, while the density in the centre is largest for short chains, giving rise to a fairly flat concentration profile. 


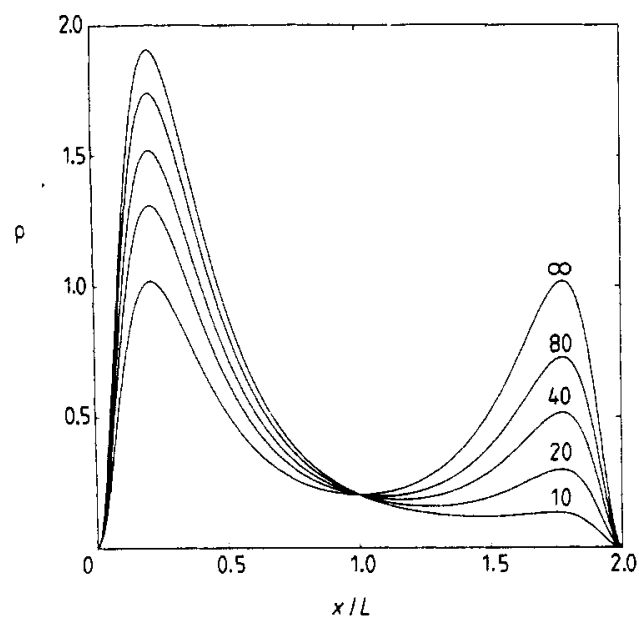

Figure 2. Monomer density between colloidal particles or cells with a Debye screened potential for tails of various length. The polymers are fixed with one end to the impenetrable wall at $x=0$, while the other end dangles free into solution. The values of the parameters as used in the text are $s_{0}=3, k_{\mathrm{D}} L=10$ and the different lengths are given by $N=10,20$, 40,80 and $\infty$. In the limit of infinite chain length all end effects disappear and there is no distinction whatsoever between tails, bridges or free polymer chains. One observes that for shorter chains the polymer material is more confined in the potential well near the left wall to which the tails are attached, while for the long chains the concentration profile is more symmetrical.

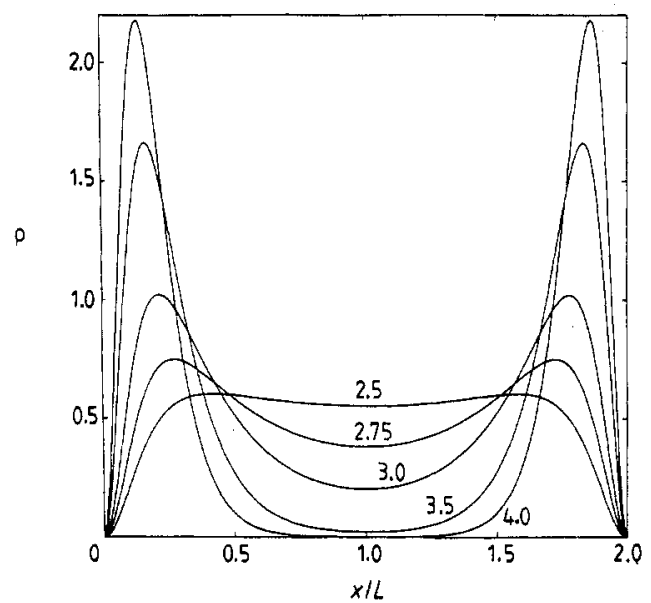

Figure 3. Monomer density between the walls in the limit of infinite chain length $(N=\infty)$ for various values of the depth of the potential well, or rather the parameter $s_{0}$, which is a combination of several physical variables (equation (12)). The values are $s_{0}=2.5,2.75$, $3.0,3.5$ and 4.0 and the particle distance is fixed at $k_{\mathrm{D}} L=10$. For small values of $s_{0}$ the monomer density in the centre is quite large. The flat profile is caused by the fact that, for shallow wells, bridges, i.e. configurations with strands of polymer material crossing from one well to the other, become more abundant. For larger values of $s_{0}$ the monomers are confined to the wells, with large strands in a single well, and only few bridges. Hence the density in the centre drops, while the maximum of the concentration curve shifts towards the walls. 


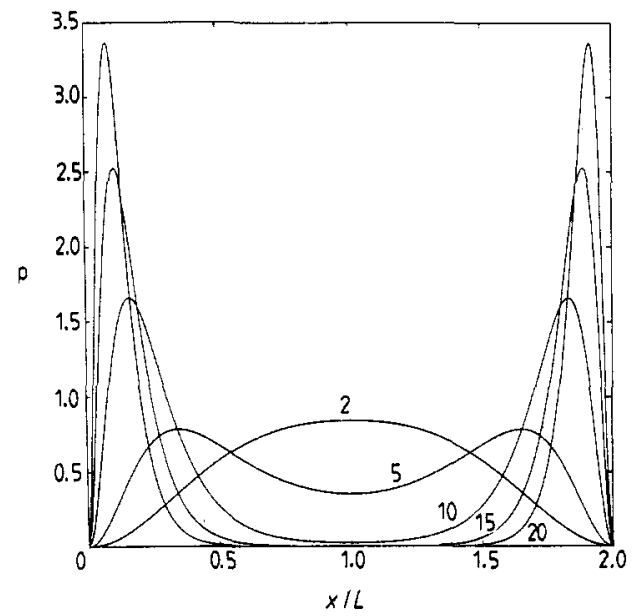

Figure 4. Monomer density between the walls in the limit of infinite chain length $(N=\infty)$ for various values of the distance between the cells or colloidal particles. The values as used are $k_{\mathrm{D}} L=2,5,10,15$ and 20 , while the value of $s_{0}$ is fixed at $s_{0}=3$. For small values of $k_{\mathrm{D}} L$ the monomer density in the centre is large. Because the particles are very close, bridges are common. In fact, for the shortest distance $k_{\mathrm{D}} L=2$, the effect of the electrostatic attraction gives rise to only a very minor broadening of the density profile as compared to that of an infinite polymer chain confined between two hard walls. For larger values of $k_{\mathrm{D}} L$ the monomers are concentrated near the walls and only an incidental bridge is formed. The shift of the maximum of the concentration curve towards the walls is caused mainly by the scaling, the value of $k_{D} L$ increases, but the width of the potential well remains fixed at about $x=1 / k_{D} L$.

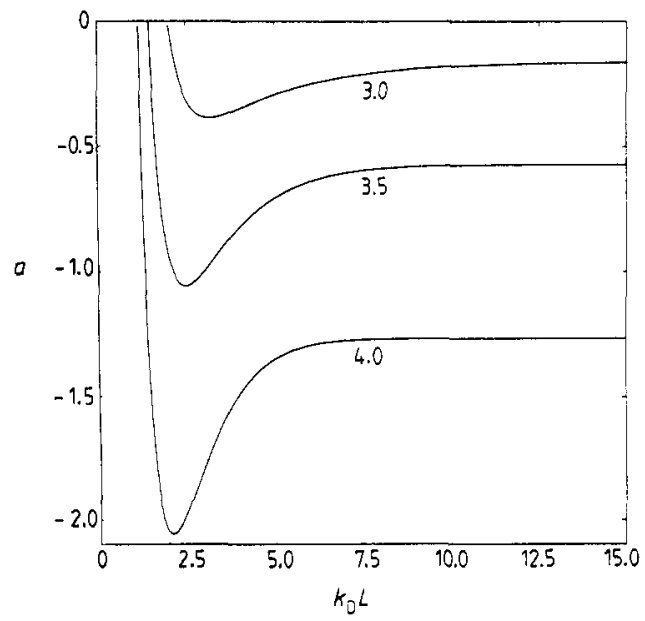

Figure 5. The free energy per monomer as a function of the distance between the two charged colloidal particles or biological cells for different values of the adsorption parameter $s_{0}\left(s_{0}=3.0,3.5,4.0\right)$. We show only the thermodynamic limit of infinite chain length as the correction due to finite chain length is only very small. One observes a minimum of the free energy corresponding to an equilibrium distance between the walls due to the effect of the polyelectrolytes. This minimum shifts towards a smaller equilibrium separation distance at larger values of $s_{0}$. The increasing depth of the effective potential gives rise to a stronger binding between the particles or cells, and hence a more stable association. 
the walls as the polymer chain starts to form an increasing number or bridges, i.e. strands of polymer which cross from one potential well to the other. Also a shift of the maximum of the curves away from the wall can be observed corresponding to a shift of the maximum of the Bessel function for decreasing values of $\nu_{s}$. For the lowest value considered, $s_{0}=2.5$, only slightly above the critical value of $j_{0,1}$ (equation (11)), $\nu_{s}$ is almost zero, but positive, and a bound-state solution is found. The density between the walls for this solution is almost completely constant. The same effects can be observed for a decreasing distance between the walls. The shift of the concentration maximum, however, is mainly due to the scaling of the abscissa of the figures, where we have plotted $x / k_{\mathrm{D}} L$. Apart from this somewhat artificial effect there is also a real shift in the value of $\nu_{s}$, as in figure 3 , but that is much too small to be observable (see figure 5). For small particle separation the monomer density again becomes very flat, because the bridges become more abundant.

\section{Thermodynamic quantities}

In order to find the thermodynamic quantities of the system we have to evaluate the full partition function

$$
Z=\iint \mathrm{d} x_{0} \mathrm{~d} x_{N} G\left(x_{0}, x_{N}, N\right)
$$

For a chain with free ends, in our approximation only the ground state contributes to the partition function; because of the antisymmetry of the first excited state. That means that the difference between the finite and infinite chain only appears in the second excited state, which we neglect. One may also observe that this is the partition function for a single chain only. But because the end effects are unimportant, one may think of the separate chains as being linked together at their ends. The total monomer density enters the definition of $Z$ only through the normalisation condition equation (29). The result is

$$
Z=\exp \left(-\lambda_{\mathrm{s}} N\right)\left(\int \psi_{\mathrm{s}}(x) \mathrm{d} x\right)^{2}
$$

from which we can easily obtain the thermodynamic quantities of interest.

The free energy per monomer $(f)$ is given by

$$
f \beta=-\log (Z) / N \approx \lambda_{\mathrm{s}}
$$

with $\beta^{-1}=k_{\mathrm{B}} T$ as before, and the pressure due to the presence of the polyelectrolytes is given by

$$
p=-\partial f / \partial(2 L) \approx-\partial \lambda_{\mathrm{s}} / 2 \beta \partial L
$$

where $2 L$ is the distance between the particles. The average internal energy per monomer is derived from the density as given in the previous section,

$$
u \beta=\int \rho(x) V(x) \mathrm{d} x=\int \psi_{\mathrm{s}}^{2}(x) V(x) \mathrm{d} x
$$

and the entropy per monomer is given directly by the relation between the other thermodynamic parameters

$$
s / k_{\mathrm{B}}=u \beta-a \beta=\int \psi_{\mathrm{s}}^{2}(x) V(x) \mathrm{d} x-\lambda_{\mathrm{s}} .
$$


In figure 5 we plot the free energy of an infinitely long polymer chain between two walls as a function of the distance between the walls. One observes that the free energy reaches a minimum, corresponding to an equilibrium distance between the particles. At the equilibrium distance the pressure, which is plotted in figure 6 , is identically zero. This is the main difference between this model, with a continuous potential between the monomer and the walls, and a short-range interaction as for instance for the lattice model we considered in an earlier paper (van Opheusden et al 1985). For short-range interactions the effective force between the plates due the polyions does not give rise to an equilibrium association distance. In the calculation of the free energy as well as the effective pressure on the plates we have taken into account only the effects of the polyelectrolyte material. At large distances this force is always attractive when there are bound states for the single-wall potential well, i.e. when inequality (11) is satisfied. When the particles approach each other the bound-state solutions disappear, and only the free solutions remain. We have not treated these solutions for the double Debye potential, but it suffices to mention that one always obtains a repulsive force due to the polyelectrolytes.

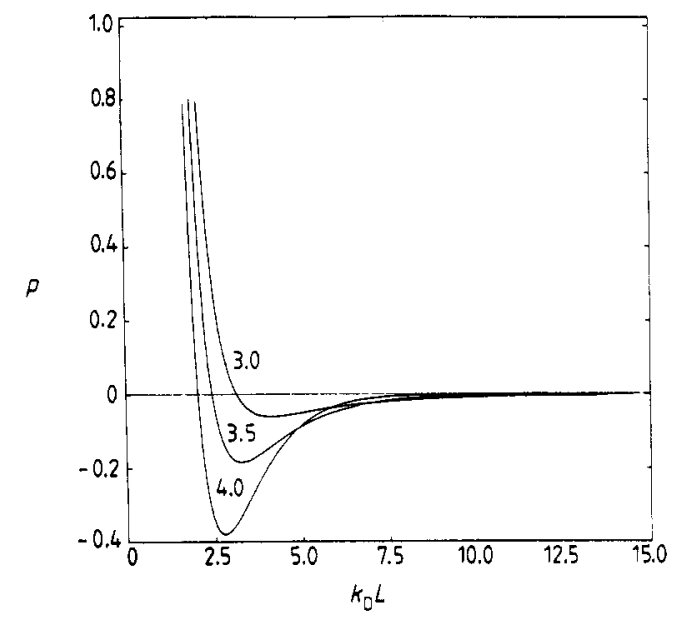

Figure 6. The pressure between the cells or colloidal particles as a function of their distance due to the presence of the polyelectrolyte material. For large distances the force is always attractive, and if the density of polyelectrolyte is large enough to overcome the direct electrostatic repulsion between the cells this gives rise to clustering or coagulation. One may note that for small distances the attractive force increases as $s_{0}$ increases, giving a more stable association, but for large distances the opposite is the case. If the particles are far apart only very few bridges of polyelectrolyte strand are formed and the coils are mainly confined to the potential well near the walls. Hence there is not much effective interaction between the walls and the attraction diminishes.

Apart from the pressure due to the polymer chains there is also the direct electrostatic interaction between colloidal particles or red blood cells. This interaction is screened by the ions in the solvent, but still gives rise to a repulsive effect at all distances, as the particles or cells have equal charge. Whether or not coagulation due to bridging of polyelectrolytes will occur depends on the competition between these two opposing forces. According to our theory, coagulation can always be inferred by adding more polyelectrolyte material, because the force which we have calculated is the force per monomer and the theory is completely linear. When, on the other hand, the monomer 
density becomes too large it is not appropriate any more to neglect the excluded volume effects of the polymer chains. It is not clear which approach in that case can lead to. results like the one described in this paper. It is possible for a simple square well to find the partition sum of an infinitely long polymer (Dolan and Edwards 1974, 1975). In the case of ground-state dominance one obtains a non-linear diffusion equation (Freed 1972) which can be solved exactly (Gerber and Moore 1977). For other potentials and, more interesting in the context of this paper, for polymers of finite length, the problem remains unsolved. The excluded volume will always give rise to a repulsive force and hence a smaller total effective attraction between the plates.

\section{Acknowledgments}

The work described in this paper is part of the research program of the Stichting voor Fundamenteel Onderzoek der Materie (FOM), which is financially supported by the Nederlandse Organisatie voor Zuiver-Wetenschappelijk Onderzoek (ZWO). Part of this work was performed during the 1986 Biophysics Workshop of the Aspen Center for Physics.

\section{References}

Abramowitz M and Stegun I A 1965 Handbook of Mathematical Functions (New York: Dover)

Dolan A K and Edwards S F 1974 Proc. R. Soc. A 337509

1975 Proc. $R$. Soc. A 343427

Gerber P R and Moore M A 1977 Macromol. 10476

Freed K F 1972 Adv. Chem. Phys. 221

Jahnke E, Emde F and Lösch F 1966 Tables of Functions (Stuttgart: Teubner)

Lampert M A and Crandall R S 1980 Phys. Rev. A 21362

van Opheusden J H J, de Nijs J M M and Wiegel F W 1985 Physica 134A 59

Verwey E J and Overbeek J Th G 1948 Theory of the Stability of Lyophobic Colloids (Amsterdam: Elsevier)

Wiegel F W 1975 Phys. Rep. 1657

1977 J. Phys. A: Math. Gen. 10299

Wiegel F W and Perelson A S $1981 J$. Theor. Biol. 88533 